\title{
My Appreciation of Geometry
}

\author{
A talk delivered at Academia Sinica, Taipei, on December 31, 2011
}

\section{Shing-Tung Yau}

\author{
Department of Mathematics, Harvard University
}

Geometry is a subject which has permeated my entire career in mathematics. I first learned the Euclidean geometry in 1961. I have worked on various problems in geometry till now. What I will talk about in this lecture is not just the Euclidean plane geometry. Its contents, methods, and results are rather deep. Modern geometry is a lively topic in mathematics. I will enlighten the audience on some history of geometry with emphasis on the concepts of harmony and symmetry. I hope to share with you the great joy that geometry has bestowed upon me.

\section{Geometry in Ancient Greece}

The ancients observed a variety of distinctive geometric patterns in daily life, which are, in general, quite transparent in character. In spite of the inherent simplicity of objects such as circles or triangles, they do, in fact, satisfy several rules. These rules are very concise, genuinely straightforward, and brimming with aesthetic appeal. The geometric problems which captivated early Egyptian and Babylonian societies - and which are found within ancient Chinese works exemplifying China's budding scholasticism, such as The Nine Chapters-were encompassed by these objects and the rules governing them. Nearly all of these important features of geometry had been explained clearly by the Greeks before 300 BCE.

\section{Thales and Pythagoras}

The first known philosopher to cast his gaze upon mathematics was Thales, a Grecian scholar who lived between 640 BCE and 550 BCE. He took the lead in utilizing logic and reasoning in order to explain and study nature, in stark contrast to the appeals to occultism and religion that characterized his predecessors. The work of Thales marked the beginning of the scientific thinking that would later pervade much of human culture.

The early successes of Thales were soon followed by a mysterious group of philosophers known as the Pythagoreans. In high school we have all encountered the remarkable Pythagorean Theorem of plane geometry, and it was this result's namesake, Pythagoras, who founded this furtive group. The Pythagoreans, whose activities spread across nearly a century, gradually formed under the guidance of Pythagoras and his many students. It was with them that one of the most important concepts in mathematics arose, the notion of "proof."

This school had a crucial influence on both philosophy and mathematics. They believed that numbers entail everything in nature, and that it is through the numbers that all things can be explained. They also believed that the universe consisted of two entities: the Number (which the Pythagoreans referred to as "perion," after the work of Thales' student Anaximander) and Infinite Space (or "aperion," literally meaning "without limit"). The Number is a finite, bounded entity, and Infinite Space is its boundless counterpart. It is through the interaction of these two substances, a process called "inhalation," that an undifferentiated whole is separated into the concomitant features of the world that we all know. Although these ideas had their roots in the Pythagoreans' cosmology, they would ultimately deeply influence the subsequent development of geometry. In fact, a staggering one thousand years later, in 1637, the appearance of Descartes' use of a coordinate system to describe geometry retained the essential spirit of these ideas. His coordinate system, which would quickly be established as the way to describe all geometric figures from then on, allows one to use numbers and algebra in order to prove a wide spectrum of geometric theorems. The points are representations of numbers, and they are located within an infinite space, which clearly parallels the notions of perion and aperion. In addition to this, other Pythagorean ideas would come to closely align with modern perspectives on the nature of reality: for instance, the Pythagorean belief that the distribution of objects affects the infinite space in which they are embedded; this is remarkably similar to the picture painted by the modern theory of general relativity, one of the hallmarks of the tremendous career of Albert Einstein. 


\section{Plato}

Perhaps one of the most influential forces for geometric enrichment would come soon after the Pythagoreans: Plato. Plato is universally recognized as a great philosopher, and he felt that geometry was the most important subject in one's education. In 387 BCE, he founded a school nestled in the suburbs of Athens called the Academy, which partially remains to this day. Legend

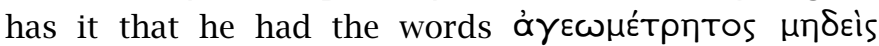
Eioítw ("Let no one ignorant of geometry enter here") inscribed above the entrance to the Academy. This epitomizes the immense importance that Greek scholars attached to geometry during this epoch.

Plato was a prolific writer, and in his dialogues he generated a vast collection of thoughts on mathematics and the universe. Of particular interest to the story we are now unraveling is found within the Timaeus: there exist exactly five regular polyhedra in 3-dimensional space, now known as the Platonic solids. This is an outstandingly gorgeous and important theorem. Even today, we are still surprised by this conspicuous statement. Plato was so enthralled by this theorem, that he even used these polyhedra to structure his cosmology.

The classic work of Euclid, Elements, provides a proof to this theorem, which underscores how familiar the Greeks already were at that time with the notion of proving theorems. Perhaps it is surprising that, in modern times, even students with a mathematics major in college may not be able to prove this classification of regular polyhedra. The proof of this theorem found in Euclid's Elements did not come from Euclid, but rather it was outlined in another text, Teaetetus, which may be, in fact, the outcome of the cooperative efforts of a group of mathematicians. At any rate, Euclid's impact would prove so significant that we will revisit this great thinker in more detail in the next section.

Returning to the Platonic solids, this theorem generated intense excitement amongst the philosophers of the age, in light of its elegance and beauty. The attraction of this result was so strong that it still lingered in the 16th century, a time when the celebrated astronomer Johannes Kepler's attempts at explaining both the structure of the universe and the operation of celestial bodies were built upon the perceived importance of the Platonic solids. Although it has since been discovered that his opinion was wrong-that the regular polyhedra cannot, in fact, be used to explain the dynamics of celestial bodies-yet the polyhedral structure has persevered in other aspects of nature: for example, in the crystalline structures that we have since observed in nature. In fact, Plato's regular polyhedra would eventually enjoy a deep significance in numerous different branches of mathematics during later days.

In addition to the regular polyhedra, Plato posed three geometric problems, which are potentially the most famous questions of the early history of mathematics, and which were likely pondered before Plato, though we do not have clear records of who was the first to put them forward:

\section{How can we trisect an angle?}

2. How can we construct a square whose area is the same as the unit circle?

3. How can we construct a cube, whose volume is twice that of the unit cube?

Each of the above questions has enjoyed an illustrious and ancient history, and their solutions have had effects which reverberated through the next two thousand years of mathematics. It is also of note that the third question had been considered in both India and China. The Greeks had already developed solutions to each of the three questions using complicated equipment; however, Plato insisted that one should strive for solutions using only the simplest geometric methods. This meant that the construction of geometric figures should involve only compasses and a ruler. Over many centuries, mathematicians passionately upheld that this simplicity should be the guiding principle when answering beautiful, foundational questions such as these. This dictum of Plato would prove to be remarkably valuable.

It is worth mentioning that the third question is firmly rooted with a legend about the ancient city of Delphi. According to the Greek historian Plutarch, in order to remove the plague inflicted upon them by the sun god Apollo, the local residents of the city of Delos in Greece pleaded with the oracle at Delphi for help. The oracle responded by requiring them to double the volume of the cube on the altar to Apollo. Perplexed by this challenging construction, the people turned to Plato for advice.

These three questions remained unsolved until the 19th century, but their solutions would ultimately play a key role in the history of mathematics. On March 30, 1796 Gauss wrote in his math diary that he had found a geometric method to construct a regular polygon with 17 sides, by using ruler and compass only. Gauss was 19 years old then, and this discovery was considered to be a turning point in his mathematical life with regard to geometry, algebra, and number theory. (It was said that before he died, Gauss demanded a geometric figure of the 17 th regular polygon to be carved on his tomb stone.)

The question of constructing a regular polygon with $n$ sides can be reduced to the case when $n$ is a prime number. In order for such regular $n$-gon to be geometrically constructed, Gauss observed that $n-1$ should be a power of 2, namely $n=2^{k}+1$ for some integer $k$. For example, $n$ can be $3,5,17,257,65537$, etc. Since $n$ is assumed to be a prime number, $k$ must also be a power of 2 . Fermat thought that any $n$ of that form must be a prime number. This was found to be false by Euler who pointed out that 4294967297 is divisible by 641 .

The work of Gauss influenced Abel, who along with Ruffini, discovered the famous theorem that general 
polynomial equation of degree greater than 4 cannot be solved by radicals. Abel gave a sufficient condition for a polynomial equation to be solvable by radicals. The condition is that the group of symmetries of the equation (later called Galois group) is commutative. The works of Gauss-Abel then led to the great discovery of Galois (1811-1832), who in particular proved that a polynomial equation can be solved by radicals if the Galois group is a solvable group. Galois' name would be woven into the tapestry of mathematical history with the later naming of these ideas as "Galois theory." Galois, in effect, employed the method of group theory to study problems concerning algebraic equations and their solutions.

It was found by Gauss and mathematicians during that period, in order for the three classical problems to be solved, certain numbers should be constructible by ruler and compass, and they must be roots of certain polynomial equations with integer coefficients, that have a specific form. Galois' great insights allowed such problems to be successfully probed. The following statement was proved by his theory: if an equation has all roots real and if it can be solved by means of radicals, then it can also be solved by means of square roots only.

As an example, one can consider the number related to the second question. As the area of the unit circle is $\pi$, the length of edge of a square with this area must be $\sqrt{\pi}$. Similarly, in regards to the third question, the edges of a cube of volume 2 will be $\sqrt[3]{2}$. Using the results of Galois theory, one can prove that the latter number, $\sqrt[3]{2}$, cannot be the root of one of the special polynomial equations mentioned above. In much the same manner, the second question does not possess a solution because $\pi$ is a transcendental number, a class of numbers that are not the root of any equation with integer coefficients. It was not until 1882 that Ferdinand von Lindemann firmly established the transcendental nature of $\pi$. In addition to these examples, Felix Klein would describe in his book in detail how Galois theory may be used to prove that one cannot trisect an angle using just a compass and a ruler. Upon the closure of the 19th century, mathematicians had finally proven that none of these three classical questions can be solved by compass and ruler as Plato had dreamed.

One may rightly be asking: Why this is an epoch-making contribution? The answer lies in the fact that it was algebra, and its introduction through group theory into geometry, that allowed solutions to these three venerated questions to emerge after twenty centuries of effort. Mathematicians prior to this work rarely considered questions about equations when approaching plane geometry and consequently failed to pay attention to the relationship between equations and geometry. Now, following this momentous achievement, mathematicians a mere two centuries ago had begun to pay attention to the importance of this burgeoning alliance.

\section{Euclid}

Euclid appeared after Plato. Euclid began with the design of five axioms and managed to deduce from them an exceptional number of interesting geometric propositions. In fact, he discovered that all plane geometry propositions known at the time could be deduced from these five simple axioms. This was a profoundly important conclusion. Mathematicians in China and India did not recognize the awesome might of axiomatization; it was only the Greek mathematicians who uncovered its importance. The deductive method allows mathematicians to deduce complex theorems from simple axioms. Following the arrival of Euclid's Elements and the presence of these Elements, this powerful method could even be felt within physics, as in the groundbreaking development of Newtonian mechanics centuries later.

Isaac Newton's epochal work The Mathematical Principles of Natural Philosophy was written in much the same way as Euclid's Elements. Newton introduced three fundamental laws, and was able to deduce the regularities of the movements of the heavenly bodies using the machinery of calculus built upon the foundation provided by these laws. This demonstrates the incomparable power wielded by the beauty of applications of logic.

In addition to this already far-reaching consequence, Albert Einstein's view on physics was also keenly influenced by the Euclidean idea of axiomatization. He hoped that all force in the universe could be explained by unified principles.

The affinity for deductive reasoning within the ancient Greek society is manifested in a variety of ways. Indeed, Euclid's ideas were, in fact, closely related to the philosophy of Aristotle. Aristotle, a student of Plato, proposed the syllogism, and contended that the deductive method consisted of three pieces: the major premise, the minor premise, and the conclusion. He utilized predetermined postulates that were inferred from our intuition as a foundation, which is called the hypothesis in English. Aristotle collected materials, derived contradictions, employed the law of the excluded middle, and then summarized and arrived at his conclusions. This process-the use of inductive and deductive reasoning, as well as their contemporary companion, abductive reasoning-is a very important aspect of the modern scientific enterprise. And it was with this foundation that Euclid was able to take his five axioms and derive all of the theorems of plane geometry.

\section{The parallel axiom}

The reasoning methods underlying plane geometry, as these embody a truly vital component of attaining any form of knowledge, should be studied by anybody who likes to apply logic in their thinking. We can uncover the importance of this statement by continuing this account of the history of geometry. 
The fifth axiom of Euclidean geometry is called the parallel axiom. It says that if a point lies off a given straight line, then there is only one straight line which passes through this point and is disjoint from the original line. If two distinct lines never meet each other, we refer to these two lines as parallel. This axiom may appear obvious; however, it was not accepted by geometers, who had attempted to prove it using the other four Euclidean axioms, but failed.

Up through the late 18th century and early 19th century, mathematicians were still adamantly trying to prove the fifth postulate; the renowned mathematician Adrien-Marie Legendre was perhaps the most notable. Legendre put a great deal of energy into attacking this problem, but ultimately failed. It was not until the early 19th century, with the introduction of hyperbolic, non-Euclidean geometry, that Klein and Hilbert succeeded in proving that the parallel axiom cannot be deduced from the other axioms, a feat achieved after an intense discussion between a few mathematical masters.

From the logical point of view, one indeed finds that not all axioms can be deduced from other axioms, which is undoubtedly a very important insight. What is more important, however, is that the concept of parallel lines, or parallel translation, that is obvious within the context of plane geometry, becomes an unexpectedly important feature of the very foundation of geometry. After two thousand years, this insight would arise and deeply affect the development of modern mathematics, and even physics. Geometers use the idea of parallel translation to illuminate the hidden inner symmetry of objects, which, for instance, lies at the heart of the symmetry principle in gauge field theory, from high-energy physics which dictates the laws of all known particles. Only with parallel transportation, we can compare objects in different position and talk about relativity.

To highlight the significance of the discussion above, consider Figure 1. There is a closed curve on a spherical surface starting at, and also ending at the vertex A. Now, we transport a vector along this closed curve, assuming that the vector remains parallel as it moves along the curve to vertex B, then from B to C, and finally back down to A. In spite of the parallel motion of the vector during its wanderings along the closed curve, we are left with a different vector when it comes back to the original vertex. The vector rotates by an angle after moving along the closed curve. The existence of this rotation angle between the two vectors indicates that it is part of the rotation group. When we push the vector along different closed curves, we are likely to get different rotation angles. It turns out that the collection of all rotation angles produced by pushing this vector along all possible closed curves forms a group, which is a subgroup of the rotation group that may be called the group of inner symmetry. One can intuitively see that this rotation is induced by the curvature of the surface involved, and so the inner symmetry group aids geometers in learning about the geo- metric structure of curved spaces. This is clearly paramount to understanding geometry as a whole. The notion of parallel transport along a closed curve may seem quite simple, but understanding it is absolutely crucial, as this examples shows. It affects the development of geometry.

Here we can see that mathematicians possess the ability to create their own world. In order to understand the idea of parallel transport, they persisted in intense research and enjoyed plentiful achievements throughout nearly two thousand years of dedicated work. This demonstrates that mathematicians are still capable of uncovering the truth that nature wants to present us with,

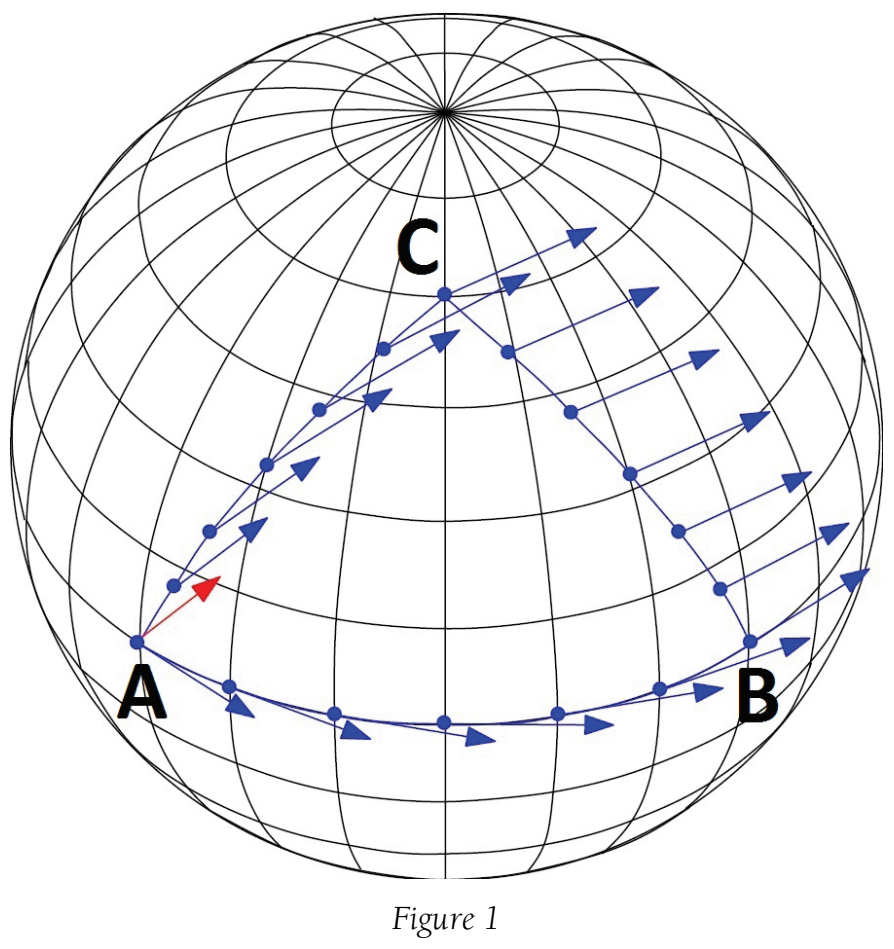

although they are armed only with their philosophy and their technology, without the aid of experiments. Sometimes they achieve the goals even earlier than physicists! Indeed, the parallel axiom led mathematicians to the idea of parallel transport, which in turn brought about the concept of inner symmetry, and this was ultimately developed into the gauge field theory.

In the above story, we witnessed the communication between mathematicians and nature. I would like to reinforce the power of this conversation by ending this section with a quote from one of the greatest mathematicians, Joseph Fourier (1768-1830). In his book Theorie Mathematique de la Chaleur, he wrote:

Mathematics can be used to determine the most general phenomena, and this is our own dominion. Since mathematical analysis determines the most diverse relations and measures time, space, forces and temperature, it is safe to say that it is as wide and rich as Nature itself. It always follows the same paths and gives the same interpretations, in that way certifying about the unity, simplicity and stability of the universe. 
Mathematics has developed in parallel with physics, chemistry and many other disciplines. But mathematicians have their own dominion, from which they certify about the unity, simplicity, and stability of the Universe. ${ }^{1}$

\section{The Spirit of Harmony}

Euclid used simple principles to interpret complicated concepts, which affected a tremendous influence on the development of art. Scholars with such a viewpoint believe that the common principles which govern nature must be concise. As a result, paintings depicting nature, as well as po-ems ex-

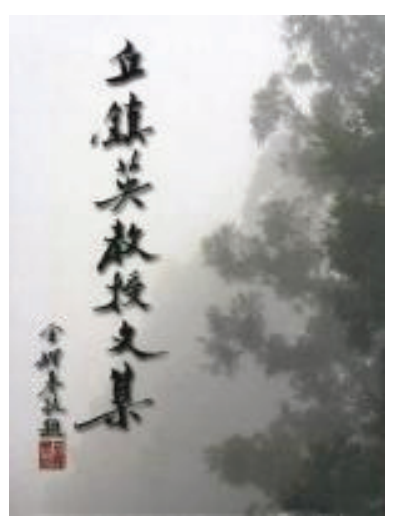
pressing the emotions elicited by nature, should be both concise and graceful. I first experienced this concept stemming from the philosophical spirit of the Greeks while I was a child reading my father's book The Complete Works of Professor Qiu Zhenying, published by the Chiao-Tung University Press, Hsinchu.

What is this Greek spirit?

The famous English writer Goldsworthy L. Dickinson, in his book The Greek View of Life, wrote: "A harmony! And in this word, we have the key to the dominant idea of the Greek civilization!"

The Greeks believed that the beautiful and the good, the body and the soul, the individual and the country, and the divine and the human being are all in harmony with each other:

1. The Harmony Between the Beautiful and the Good: Plato mentioned in The Republic: "Let our artists rather be those who are gifted to discern the true nature of the beautiful and graceful; then will our youth dwell in a land of health, amid fair sights and sounds, and receive the good in everything." ${ }^{2}$

2. The Harmony Between the Body and the Soul: The body is capable of expressing the truth encompassed by beauty, just as the breeze from the Holy Isle of the God can unconsciously arouse a sense of beauty and compassion within our soul. This is how Plato viewed the harmony between the body and the soul.

The Greek statesman Pericles said, "For we are lovers of the beautiful, yet simple in our tastes, and we cultivate the mind without loss of manliness." Which means that a beautiful soul is housed in a beautiful body.

\footnotetext{
${ }^{1}$ M. A. Akivis and B. A. Rosenfeld, Élie Cartan (1869-1951), AMS, 1993

${ }^{2}$ Translation by Benjamin Jowett, Wildside Press, 2008, p. 109
}

Plato suggested that music and gymnastic are the two divisions of education, gymnastic for the body, and music for the soul, ${ }^{3}$ and that we should strive for the harmony of body and soul.

3. The Harmony Between the Individual and the Country: Aristotle described a state as "a community of equals, aiming at the best life possible." ${ }^{4}$ So the Greeks never attempted to elude the compulsory duties to their country, but at the same time continuously chased after their freedom. It was in this way that they maintained the harmony between the individual and the country.

4. The Harmony Between the Divine and the Human Being: The Greeks believed that the divine is beautiful and pure, and so gods are always depicted as charming and goddesses are always chaste. This exemplifies the ideal of the Greeks. It is not a mere appeal to the superstitious when they try to express their thoughts and dreams through the divine.

As we have seen, the concept of harmony is one of the most important aspects of the Greek civilization.

The relevance of the idea of harmony outlined above can be found in the evolution of ancient mathematics into its modern form. It is for this reason that we should take seriously the basic thoughts of these eminent Greek philosophers.

The beauty of mathematics brings us much closer to nature. Nature helps us to open our minds and broadens our visions. This clearly explains why there are geometers involved in research on every topic from the origins of the universe to the planetary motion, and from the structure of the atom to paintings of nature. They delve into nature and make tremendous contributions.

Geometry dominates a diverse spectrum of fields. In order to appreciate this, one should note that other factors, apart from the interest in aesthetics, also influence the development of geometry.

For example, no matter which of them you examine-be they from Egypt, Babylon, India or China-the ancient scholars all had a strong inclination towards the study of the calendar. We should note that these countries were based on agriculture, which led them to devoting much effort to researching the movement of the planets. Each of these had enormous influence on the development of geometry.

The ancient Greeks had already figured out how to measure the radius of the earth and how to approximate the distance between the earth and the sun. By this time, they had discerned that the earth is round. The ancient Chinese initiated great contributions to mapping the earth, as they were fascinated by the study of the geometry of the earth, as well as of the heavens. In the early

\footnotetext{
${ }^{3}$ Ibid., p. 76

4 Translation by Benjamin Jowett, Random House, New York, 1943, p. 293
} 
years of the Han dynasty, Liu An, a ruler of the Huai-Nan county, wrote a book entitled Huai Nan Zi, in which he outlined a method for measuring the distance between the earth and the sun. The method of measurement contained in that book is essentially the same as what the ancient Greeks employed. Another example is that of the calendar. It is believed that calculation for the calendar was the source of the discovery of the Chinese remainder theorem. These two examples clearly illuminates the significant role that curiosity, as well as the demands of daily life, played in the ancients interest in studying geometry.

There is no doubt that the overall advancement of geometry over the past two millennia was based on the idea of harmony, which fascinated the ancient Greeks so much.

The Pythagoreans thought that music can be explained by numbers. This might seem to have nothing to do with geometry, but the famous research on wave mechanics carried out by Fourier in the 19th century demonstrated that all waves are made of basic waves, and this opened the door to the field of spectral analysis. We now know that every geometric figure has its own spectrum. The study of spectra in geometry encompasses the main trend in today's geometry, and spectra are simply a series of numbers to mathematicians, which is the gist of what the Pythagoreans had envisioned.

As an example, consider what happens if a geometric object is made into the frame for a drum, and this drum will produce sound waves when beat. We can study the sound waves through spectral analysis of the audio frequencies. The spectral analysis may allow us to infer the geometry of the drum. If we beat an oval drum, as opposed to a round one for example, the sound waves produced will themselves be sufficient to judge whether or not the drum is circle. Were we to construct a rectangle drum, we could also interpolate the spectral data and find that the spectrum is not the same that of the oval one. This prominently highlights how deep is the connection between the sound waves generated by the drum and its geometry.

The beauty of music can be perceived by our auditory system and the beauty of geometry can be discerned by sight. This great harmony that lies within the beauty of music and geometry can be rigorously defined by mathematics. Fixing the two ends of a string, one can set it vibrating and it will produce a diverse range of sound waves, which can then be expressed by the sum of sine functions with different frequencies. One sees a beautiful picture when we draw the graph of the function $\sin \frac{n x}{L}$, which represents one of the basic waves. What is worth mentioning about this example is that the spectrum of the sine function referred to above is $n^{2} / L^{2}$, where $n$ is any natural number. This reveals that the knowledge of the spectrum allows one to determine the length $L$ of the string.
There is an important class of functions defined on the space found in the theory of complex functions, which epitomize the role of harmony within geometry and demonstrate how harmony can be expressed via mathematics. These functions, known as harmonic functions, satisfy an important property: the value at each point is equal to the average value on any sphere surrounding it. In other words, it might be said that these functions possess what the ancient Greeks referred to as the property of the Middle Path, and is equally represented by the conception of harmony put forth in the Doctrine of the Mean of Confucius, the ancient Chinese sage.

In the above, we mentioned that, when one beats a drum, the vibrations of the drumhead are composed of a combination of basic waves. The basic waves have expressions that bear striking resemblance to the notion of harmonic functions. It reveals a deep commonality between music and painting: both can be understood in terms of basic elements that are elegant.

In fact, these waves encode their own physical meaning: every wave has its specific energy. Each basic wave has the least energy in its state, which is a consequence of a fundamental principle both in physics and in geometry: in physics, we know that materials will be most stable when they are in their states of lowest energy. In geometry, we know that geometric figures are most elegant and beautiful when they have least length or least area.

Throughout the history, scientists have studied the vast range of natural phenomena by employing this principle. Making flexible usage of this idea allows us to explain a plethora of common phenomena, such as the fact that "water flows from high to low."

The same experience also exists within observations of humanity: under specific conditions, according to the judgment of the Highest Good, we need to reduce desire to the lowest level possible. This idea of purifying one's soul by minimizing greed and desire deeply resonates with the beauty of geometry personified by this principle. As a consequence, a harmonious balance between the beauty and the good can be achieved.

Mathematicians often prefer serene and pure matters. The influential French geometer Élie Cartan, a mentor of my advisor Shiing-shen Chern, said: "Every time I had a chance to listen to Hermite, I had before me an image of quiet and pure joy caused by contemplations about mathematics, joy similar to the one that Beethoven must have felt while feeling his music inside of himself." This quote captures the essence of the glimmering similarity in thought between geometers and composers.

\section{The Emergence of Symmetry}

Symmetry was not formalized until the 19th century; however the idea can be traced back to as early as the five Platonic Solids mentioned earlier. Indeed, why are we in- 
terested in regular polyhedra? The answer lies in their good symmetry properties.

The Greeks first discovered four polyhedra composed of triangles and squares (i.e., excluding the dodecahedron). The Greek philosophers ascribed to them some physical meaning: fire corresponds to the regular tetrahedron, soil to the cube, gas to the regular octagon, and water to the regular icosahedron. It filled them with joy to have felt that they uncovered the geometry underlying the four basic elements of the universe.

It was soon after, however, that they found the regular dodecahedron unexpectedly. This particular polyhedron proved much more difficult to discover than the others in light of its composition in terms of pentagons, rather than triangles and squares. The Greeks excitedly exclaimed that it corresponds to another basic element of the universe called the ether, which was thought to be the medium through which transmission occurred. This idea

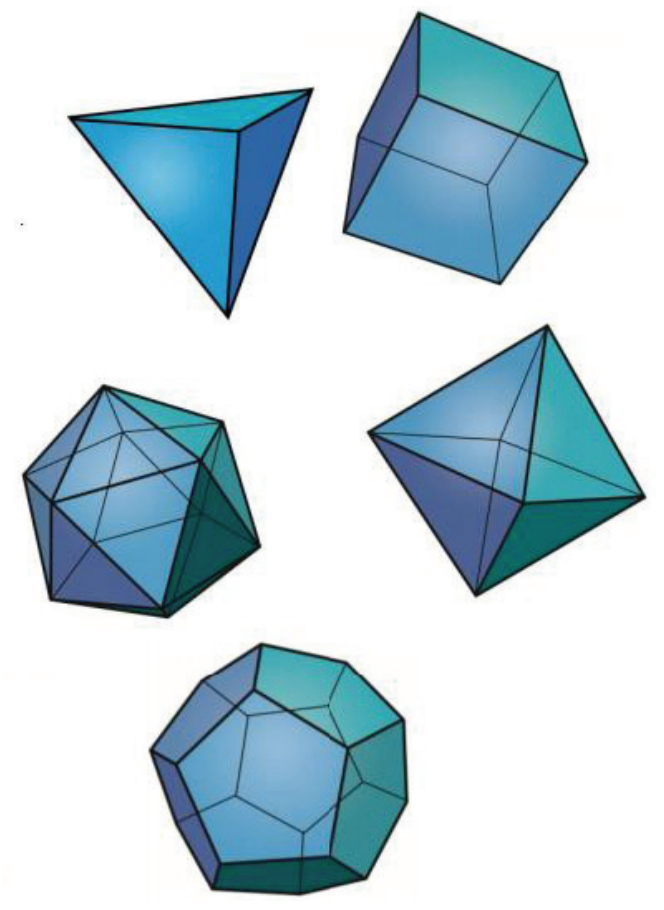

Figure 2. Polyhedra

of the ether is another concept in the long Greek litany, which genuinely impacted the development of science and would persist for centuries. Ultimately, the nonexistence of the ether was demonstrated by the famous Michaelson-Morley experiment at the end of the 19th century.

Although these explanations of the nature offered by the ancient Greeks are not accurate, the concept of symmetry underlying them, which later evolved into the idea of a group, has greatly influenced the development of physics from the beginning, and continues to do so up to this day. The Galois group briefly mentioned earlier can safely be called the beginning of this revolution. Galois was a young genius, a mathematician whose contributions influenced many great mathematicians to come, such as the Norwegian mathematician Sophus Lie, the French mathematician Élie Cartan, and the German mathematicians Felix Klein and Hermann Weyl, amongst countless others, who all devoted themselves to the study of the structure and applications of group theory. In the late 19 th century and the 20th century, the power of the symmetry group prominently displayed itself in geometry and became the support for modern geometry, and even modern physics.

Chen-Ning Yang, one of the greatest theoretical physicists who has a deep understanding of the concept of symmetry in science, wrote as follows in his essay "Einstein's impact on theoretical physics":

The first important symmetry principle discovered in fundamental physics was Lorentz invariance, which was found as a Mathematical property of Maxwell equations, which in turn were based on the experimental laws of electromagnetism. In this process, the invariance, or symmetry, was a secondary discovery. In his Autobiographical Notes, Einstein gave Hermann Minkowski credit for turning this process around. Minkowski started with Lorentz invariance, and required that field equations be covariant with respect to the invariance.

Einstein himself was deeply impressed by the powerful physical consequences of symmetry principles and worked to enlarge the scope of Lorentz invariance. This idea of a more general coordinate invariance led, together with the equivalence principle, to the general theory of relativity. We might say that Einstein initiated the principle that Symmetry dictates interactions. This principle has played an essential role in recent years. ${ }^{5}$

Indeed, Einstein himself acknowledged the profound influence of his teacher Minkowski on using the Lorentz group to put the concept of space-time together. With Lorentz symmetry, he explained the physical properties manifested within the special relativity theory, which was considered natural and well defined within Minkowski space. The result was that the concepts of time and space are inseparable. This legacy has continued until today: physicists still regard space and time as aspects of a single structure. The physics of the 20th century was profoundly altered because of the emergence of this concept of symmetry. It influences greatly both science and philosophy. Space and time have remained linked together; space is no longer just space, and time is no longer just time.

Einstein soon realized that Lorentz group was not enough if he wished to make the theory of gravity consistent with special relativity. By thought experiments, he found out that a much bigger group of symmetries is needed: the group of diffeomorphisms of the space-time.

\footnotetext{
${ }^{5}$ Physics Today, June 1980
} 
This group can be considered as the group of all possible frames of observers that may be accelerating.

This, however, is not the only place in which symmetry has made its appearance. The potent idea of symmetry often appears in the Chinese classic Yi Jing (易經). Chinese people are known to be fond of talking about "Yin" and "Yang" (the Dark and the Bright). In fact, the vast contents of the mathematics are, as well, replete with notions of duality. These ideas are yet another relic of the legacy of early Greek mathematics. The Greek mathematicians Apollonius and Pappus both conducted research on the dual relationships found within projective geometry, and in particular they discussed the duality manifested with the reciprocal concepts of "pole" and "polar." I first learned about these concepts while I was in middle school; they are, unfortunately, no longer taught in today's middle schools. Indeed, the idea of duality has a profound effect on modern mathematics: both modern topologists and modern algebraists need to utilize various incarnations of duality. It has really arisen as an important tool in modern mathematics.

During the past 30 years, we have discovered another important symmetry, called mirror symmetry, within the study of string theory. In the cosmic structures proposed by string theory, it was found that a mathematical object known as Calabi-Yau space can be used to construct a model for the vacuum state of the universe. They found that there is another Calabi-Yau space that is dual to it. In this duality, space with big diameter is dual to space with small diameter, and the quantum theory determined by them describes equivalent physical phenomena. In this way string theory uncovered a wonderful treasure: that a strongly coupled theory is dual to the weakly coupled theory associated with the dual space! This provides us with one of the most effective methods of calculating strongly coupled quantum field theory. If string theory can be verified experimentally, it would mean that the concepts of "Yin" and "Yang" are explainable within the purview of mathematics! After all, the Taoists (a traditional groups of Chinese traditional philosophers) often say that the maximum is dual to the minimum.

We mentioned before that the ancient Greeks adored manly gods and beautiful goddesses. This can be regarded as a duality between the firm and the soft. Thus we once again see that this notion of duality permeates numerous facets of human activity, from mathematics to art, and to philosophy.

In addition to this, during the Renaissance period, artists applied the skills of composition and painting to emphasize the depth of scenery, and this kind of technique enjoys a close relationship with projective geometry.

Perhaps the most prominent of Italy's architect-artists during the Renaissance were Brunelleschi and Alberti, who wrote a prodigious number of books endeavoring to express the depth of scenery. In order to achieve this, they needed to research the relationships inherent in mappings between different planes, and consequently they unearthed a plentitude of useful duality principles. This underscores the broad impact of artists upon geometry.

In fact, this is seen not only in the western scholars during the Renaissance, but also through Chinese scholars in their extensive study of the geometric properties of perspective. During the 18th century, Xiyao Nian (年希堯), the brother of the famous general Gengyao Nian (年美堯) of the early Qing dynasty, was involved in this kind of research, but unfortunately his studies did not attract the attention of other mathematicians in China.

Projective geometry greatly influenced the development of modern mathematics. It gave up the notion of distance which was so common in metric geometry. However, for the projective geometers of the 15th or 16th century, distance was not the subject of this study. They had begun to study various questions related to the intersection of lines, and of lines and planes.

Figure 3 below depicts one problem they studied. The two gray triangles possess vertices lying on the three rays protruding from the point $\mathrm{O}$, which will be labeled as OAA', OBB' and OCC'. The three sides of these triangles are given by: $B C$ and $\mathbf{B}^{\prime} \mathbf{C}^{\prime}, A C$ and $\mathrm{A}^{\prime} \mathrm{C}^{\prime}$, and $\mathrm{AB}$ and $\mathrm{A}^{\prime} \mathrm{B}^{\prime}$, respectively. Extending these sides, one finds that they intersect in three points, which we will call $\mathrm{R}, \mathrm{P}$ and $\mathrm{Q}$. The geometers of this period proved that the three points $\mathrm{R}, \mathrm{P}$, and $\mathrm{Q}$ must lie on the same straight line. With this discovery, projective geometry began to develop into the primary trend in mathematical knowledge during the 19th century. This field's influence was no longer restrained to simple drawing problems, but became the key to unlocking marvelous developments in number theory and topology.

Above we witnessed another positively significant example of how mathematicians' interest in the pursuit of beauty can also lead to the heights of performance. Freeman Dyson in his "Obituary for Hermann Weyl" writes: "My work always tried to unite the true with the beautiful, but when I had to choose one or the other, I usually chose the beautiful." ${ }^{6}$ Fortunately the truth revealed by nature is teeming with wonderful examples of beauty. Generally speaking, it is rare that mistakes or contradictions would appear in beautiful mathematical works.

Many years ago, I was a faculty member at the Institute for Advanced Study in Princeton. This Institute, where Einstein and Weyl worked, maintains a spirit of holding beauty as a sign of truth. The emblem found in the Institute's halls is richly adorned with the image of a nude goddess on the left, and of a clothed goddess on the right. One goddess represents truth and the other represents beauty. Any one, whether a writer, an artist, a musician, or even a mathematician, cannot help but pursue

\footnotetext{
${ }^{6}$ Nature, 177 (1956) pp. 457-458; also, The World of Mathematics, James R. Newman, ed., vol. 3, 2000.
} 
the meaning of beauty, nor stop chasing all conceivable methods of expressing beauty.

\section{Geometry's Intrinsic Beauty}

Different people hold different views on the concept of beauty, and consequently they will differ in their judgment of the beauty of mathematics. One may find this curious. In fact, both the concept of beauty, and the appreciation of geometry, are ever-changing with time. Within different epochs, we have different views.

As such, ancient views on beauty are not necessarily aligned with the present. For example, in the 19th century Bernhard Riemann proposed a new geometric point of view, which thrust geometry into a grand new era. Curved space was no longer a surface embedded within the Euclidean space that we experience, but an entirely

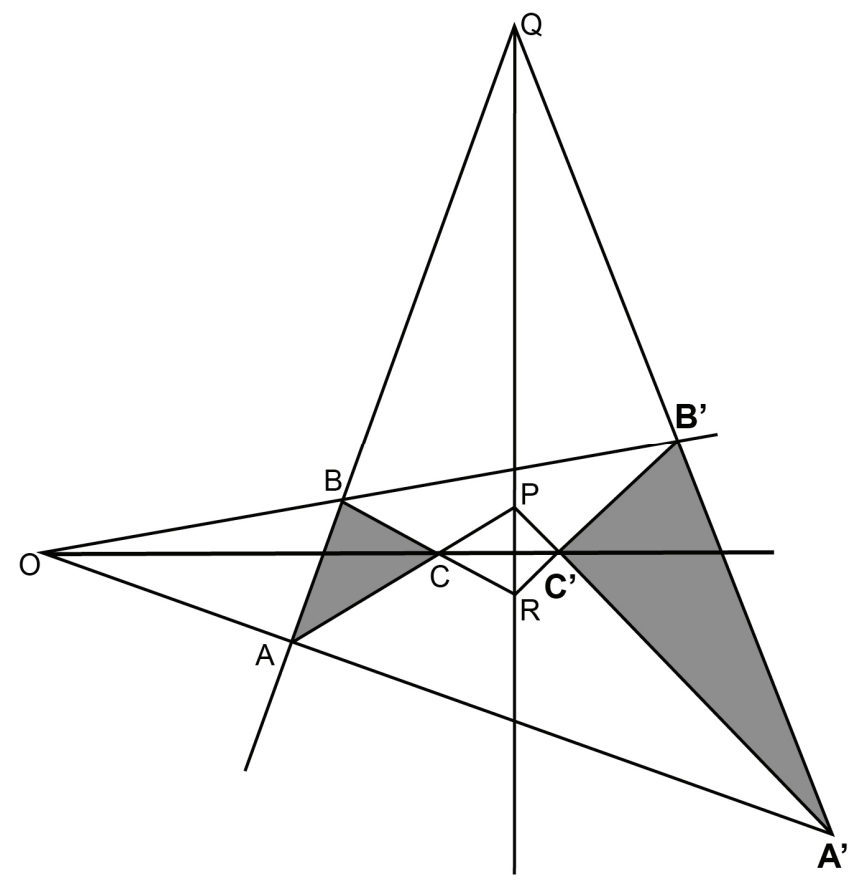

Figure 3

separate space. It has an independent significance. This marked an epoch-making contribution, because it gives the concept of the space a great degree of freedom. In Riemannian space, we can measure length, measure curvature, and even conduct differential and integral calculus-all free from relation to any ambient Euclidean space. With Riemannian geometry, we found a more in-depth understanding of space than ever before. We cannot see this class of geometric spaces with the naked eye, but we are able to calculate and measure the different geometric quantities involved.

There is a concept of intrinsic symmetry group that we can associate to a Riemannian space that is different from the concept of symmetry that can be traced back to the Greek geometers. When we saw Platonic solids, we found them pleasant. Why? It was simply because they possess a feeling of symmetry; and this resonated with us. On the other hand, general curved spaces, however, have no obvious symmetry group. This might be surprising, since we have learned that the concept of symmetry is very important. In light of this, mathematicians and geometers, who would still prefer to be guided by the notion of symmetry, began to contemplate a seemingly "internal" symmetry group of space.

During the late 19th century and the early 20th century, two well-known French geometers, Jean-Gaston

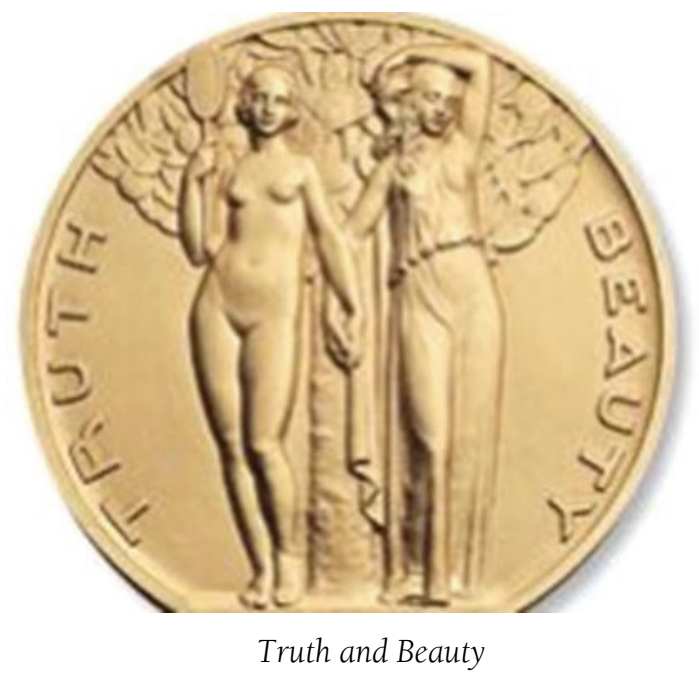

Darboux and Élie Cartan, proposed a new conception of symmetry. They introduced the concept of the holonomy group. What is this? We saw in a previous figure that there are multiple closed curves on the sphere, and along each closed curve one finds a rotation angle after parallel transporting a vector around the closed curve and back to the original vertex. We learned that these rotations form a group, which is a subgroup of the rotation group; we call this subgroup the holonomy group of the space. So, even if the space exhibits no external symmetric, we can still introduce a notion of symmetry. At every point of the space in question, we can connect group theory and geometry to each other. This group, derived from the parallel motion along closed curves, displays a close relationship with the geometry of space itself.

Internal symmetry describes the inner beauty of a given space through the holonomy group. Consequently, the classification of geometry is closely related to the classification of holonomy groups. At each point of the space under study, we can define a holonomy group, and we find that this group is independent of the choice of point. It is a breed of "Lie group."

The great mathematician Sophus Lie initiated the theory of Lie groups in the mid-19th century. It was not until the 20th century, with the work of Cartan and Weyl, that a classification of Lie groups was completed. This is surely one of the most important achievements of contemporary geometry. 
As alluded to above, the holonomy group is one type of Lie group, and therefore we can progress in the classification of holonomy groups through the work of these brilliant mathematicians. It is a manifestation of the close relationship between geometrical classification and symmetric groups, and marked a tremendous accomplishment in the field of geometry during the period from the 19th century to the 20th century. Its impact on modern geometry is far-reaching. Moreover, the arrival of appreciation for inner, rather than eternal, symmetry signifies the stark contrast in the conception of beauty observed by ancient geometers and that of today.

Aside from the relationship that global geometry shares with Lie groups, it also has deep connections with theoretical physics. In our above discussions of holonomy groups, we found that we do not require the concept of distance; one only needs to use parallel transport to define the holonomy group. This simple parallel movement of a vector along a closed curve is in no way assisted by the concept of distance. This fact served as the starting point of gauge field theory.

In modern string theory, a space called Calabi Yau space was introduced. Such space has no obvious group of symmetry. But their holonomy group, which may be considered to be "internal symmetry group," is special. It is given by the Lie group $S U(n)$.

\section{The Interplay Between Mathematics and Physics}

The application of gauge fields in physics began with the great mathematician Hermann Weyl. In his attempt to unify the theory of electromagnetism and general relativity, Weyl created this fantastic concept. In fact, the name "gauge theory" was coined by him. In 1954, Chen-Ning Yang and Robert Mills generalized Weyl's theory to non abelian gauge theory which dictates fundamental forces between elementary particles. After the complicated process of quantizing classical field theories coupled with the considerations of symmetry breaking, we arrived upon the standard model of modern particle physics. It is here that we discover that all fields are describable via gauge invariance, which is both a beautiful and important development. In reality, gauge theory produced a significant effect on geometry even earlier than it did so on physics.

However, even before the birth of modern gauges theory, the first interaction between physics and geometry in modern science is found in Einstein's general theory of relativity.

In order to describe gravity, Einstein pointed out that the space described by Riemann might change over time. The local behavior of space-time can be understood within the framework of Lorentz transformations. The Einstein field equations dictate the change of space-time, and we are forced to replace the notion of space with that of space-time. This generates a great deal of activities in both the physics and the geometry communities. Geometers took nearly one hundred years to fully study and appreciate the space that is described in Einstein's general theory of relativity. Einstein's theory points out that the gravitational field is determined by the structure of the space-time geometry. Recently when I read Riemann's old paper, I am convinced that the theory of general relativity is exactly what Riemann wanted when he formulated his concept of space. Space is no more absolute. The gravitational field brings about a change of space, while on the other hand, the gravitational field can be explained by the concept of curvature in geometry. The deduction of this theory is truly elegant. When I read Einstein's theory of general relativity, I feel as though I am viewing a beautiful classical Chinese painting.

In an address entitled "On the origin of the general theory of relativity" that Einstein gave at Mein Weltbild, Amsterdam in 1934, he said:

In the light of knowledge attained, the happy achievement seems almost a matter of course, and any intelligent student can grasp it without too much trouble. But the years of anxious searching in the dark, with their intense longing, their alternations of confidence and exhaustion and the final emergence into the light. Only those who have experienced it can understand that.

Within the space-time of Einstein, we can run into rather strange points. The curvature actually becomes infinite and the known physical laws cannot held at these points. The geometry near these mysterious points can be used to describe the objects now known as black holes. It was only within the past twenty years that astronomers began to accept the idea of black holes. Now, astronomers believe that black holes are an entirely commonplace objects. Indeed, much has changed since the time in which they were regarded as merely an abstract model invented by mathematicians.

The model of black holes includes thin, apparently invisible films encasing the singular points described above. Curiously, light within the films is incapable of escaping the region. This explains the origin of the term "black hole." The properties of the film are quite similar to those of soap bubbles-much like those we find in our kitchen sink. It is a bit strange that surfaces inside space are actually related to black holes, not to mention soap bubbles. I imagine that many people will laugh at the very mention of soap bubbles. Such a reaction is underestimating the power of these seemingly simple objects. In fact, research on the structure and properties of soap bubbles constitutes an important area of knowledge within present-day geometry. In our studies of soap bubbles, we actually uncovered the startling picture given above: the theory of surfaces arising within space is quite closely akin to the surfaces of soap bubbles.

Researching curved surfaces in space is a very important subject. In the 19th century we began to study 
families of planes. Although the plane itself is a very simple object, research into families of planes is not so simple, and so even to this day there are a lot of people devoting themselves to this area of research.

During the period from the 18th century to the early 19th century, Julius Plücker created the coordinates of families of planes, which was a great contribution. His student Hermann Grassmann, inspired by Plücker's coordinates, introduced an entirely new type of number system (the Grassmann algebra), and went on to develop the various properties of these numbers. These Grassmann numbers are quite different from ordinary numbers, primarily due to their squares being equal to zero. Grassmann numbers were eventually found to be of vital importance to describing fermions in modern high energy physics. This codified Grassmann's discovery as an invaluable tool.

Following the introduction of Grassmann numbers, the theory of exterior differential forms appeared. This important theory has influenced geometry for more than one hundred years, and has become an important bridge between topology and geometry in the hands of Georges de Rham, Élie Cartan, William V. D. Hodge, and Kunihiko Kodaira.

It is worth mentioning now that Hodge theory and the theory of exterior differential forms are also locked in a harmonious embrace. Hodge found that the exterior differential forms enjoy a close relationship with the curvature of the space on which it is defined. This British mathematician proposed a grand conjecture that would provide a means to use exterior differential forms to study algebraic manifolds. It played an important role in bringing together geometry, algebra, and topology. Moreover, it can justifiably be said to be one of the most important conjectures in all of mathematics. Although the conjecture remains unproven, it has still affected a tremendous impact on the mathematical enterprise.

Under the influence of fluid mechanics and electromagnetism, the original Hodge theory studied what would happen in a force field or an electromagnetic field under certain topological constraints. In fact, back in 19th century, Niels Henrik Abel and Bernhard Riemann had already begun to study these phenomena, which once again highlights the effect of physics on geometry.

\section{The Geometry of Static and Dynamic}

Geometry has undergone different stages of development. Geometers have spent an enormous amount of effort, and have succeeded in obtaining copious numbers of important results during the past hundred years; however, most of the work discussed thus far has involved concepts that are static in nature. Over the past twenty years or so, we have forged ahead into the study of the dynamics of geometry, which consists of questions and concepts that are significantly more profound, and more deeply difficult, than their static counterparts. As an illustrative example, I note that it is possible to produce multifarious geometric images from flowing liquid. Although the theory of fluids is quite nice in many respects, the true nature of fluid is considerably difficult to understand. Even today, we still cannot effectively grasp the turbulent changes in the liquid. When the environment or energy suffers violent changes, fluids may become quite tremulous, and therein lie numerous difficulties, even with our modern understanding of geometry.

Perhaps the most well known example of dynamic problems encountered in geometry is found in the Ricci flow theory created by my friend Richard Hamilton in the 1980s. His equations describe a specific change in the geometry of the space under consideration. Hamilton's theory is both esoteric and beautiful. By utilizing his equations, we may push the space and keep it moving continuously. In this way, we will then understand the internal structure more deeply. It is Hamilton's equations that allow us to deform the space from a complicated one to a simple one.

For instance, the surface of a dumbbell can be transformed gradually into a sphere, a process provided by Hamilton's equations. In other words, we can use these equations to propel the space into a state of constant change, culminating in an extremely simple space at the end. This idea inspired Hamilton to follow up with the completion of a wide array of other important works. One noteworthy application is made by the Russian mathematician Grigori Perelman on the famous Poincaré conjecture.

The concept of Hamilton's Ricci flow is yet another exhibition of the close relationship with the harmony described above. We know that the metric is used to measure the distance in differential geometry; the coefficients of the metric are functions of the coordinate system. When the coefficients are harmonic functions in a well-chosen coordinate system, the metric is called an Einstein metric, to commemorate the great Albert Einstein. The Ricci flow interprets the harmonic metric by a dynamic description. It provides a method to deform the metric of the space, so that it can be gradually turned into harmonic metric. The ability of Hamilton's formalism to make the metric harmonic has brought about a profound change in geometry.

Since the emergence of quantum mechanics, geometry has acquired many new tools. Among these tools, the Dirac operator is the most important one. Although it has been used to solve many important geometric problems, the emergence of the Dirac operator greatly confused geometers. Even today, its geometric significance is still not fully understood. It does, however, share a very close relationship with the harmonic functions, and the solutions of a Dirac equation can be interpreted as square root of harmonic functions.

In conclusion, the harmonic metric can be manifested in a way similar to the construction of harmonic functions. The dynamic performance is guided by the heat conduc- 
tion equation, which appears when heat changes. Hamilton's Ricci flow can be regarded as the "heat flow" over the space of metrics.

Another dynamic performance is represented by the wave equation. The Einstein equations can be regarded as the wave equation over the space of metrics. The wave equation has also a close relationship with the concept of harmonicity, and is, in fact, a dynamic representation of harmonicity.

On the other hand, we should remember that the symmetry group can also be used to expose the concept of harmony. The Platonic solids make geometers appreciate the beauty inherent in symmetry, which is manifested within these regular polyhedra. When the importance of groups in solving equations was discovered by Galois, the concept of a group was officially introduced into mathematics. Mathematicians began to understand the meaning of symmetry. Thereafter, number theory and geometry began an epoch-making change. Following that, representation theory of Lie groups and its internal discrete groups had a significant impact on the sciences in general.

\section{The Physique of Geometry}

Both Confucius and the Greek philosophers emphasized the training of the body. Aristotle maintained that the Greeks had high-mindedness, meaning that they were as proud of themselves as the stallion that can run thousands of miles in a day; they were strong human beings with very high opinions of themselves; they took pity on others, but never accepted pity from others; they owned slaves, but never become slaves themselves. By the same token, we have a saying of the famous Chinese sage Mencius: "Wealth and honor infatuate him not; poverty and inferiority divert him not; power and force intimidate him not."

Any profound science has its essence; the knowledge that is accumulated over thousands of years is the body of science. But without the essence, we scientists have no idea how to innovate. We need to innovate because that is the goal of science. And we must come to understand what is the physique-or essence-of geometry.

In geometry, we need to find out precisely what our spaces look like. In the late 19th century and the 20th century, most of our research focused on smooth spaces, although a few geometers contributed to the study of rough spaces. As an example of the latter, in algebraic geometry we conducted research into the space with singular points defined by polynomials; some geometers studied the singular points arisen from the nonlinear equations.

Unfortunately, we have little knowledge of another rough, yet real, space. This is the geometry that may be produced by a merge of quantum principle with differential geometry. Such spaces will be far more complicated than anything we know before and yet it will be essential for us to understand their nature.

Even from computer science, we can uncover interesting geometric spaces which appear in the study of large networks. When the network increases in size, we find traces of continuous geometry. All of these are the objects which should be researched further. As of yet, geometers still have no systematic methods for studying non-smooth spaces.

On the other hand, even our knowledge of smooth space is not deep enough, despite that they are the first spaces we like to understand. In such spaces, we wish to build some structure, with which we hope to perform analysis, algebra, or physical calculations.

Geometric structure over a space can be considered as the backbone of geometry. We need to determine a way to construct the foundation of geometry so that we can develop beautiful theorems in geometry. But how can we build these geometric structures? Generally speaking, the first step will require deep understanding of topological spaces.

After the combined efforts of topologists and differential geometers over the course of more than a century, we have achieved a basic understanding of the topological structure of higher dimensional smooth spaces. In many cases, we can reduce the classification of topological structures to a matter of algebraic calculation.

As an example, we can characterize the topology of the two-dimensional curved surface by the genus.

In the higher dimensional smooth spaces, there are homology groups, homotopy groups, and characteristic classes, such as the Stiefel-Whitney class, the Pontryagin class, and the Chern classes. We may classify most topological spaces by this algebraic apparatus; we may even input this information into a computer database. We now possess great knowledge of topological classification. But this is just the first step in studying geometric structures.

How can we construct the geometric structures systematically? So far, the main technique is to use algebraic method or differential equations to generate a geometric structure.

The first successful example is the discovery of Riemann surfaces, which are two-dimensional surfaces that affected a powerful change in geometry. The theory of Riemann surfaces was initiated by Riemann in the 19th century. It continues to play an important role in modern mathematics.

For three-dimensional manifolds, my classmate William Thurston initiated a very important direction of research in the 1970s, namely, the study of geometrization for three-dimensional manifolds. The final steps of Thurston's ideas were supposed to be completed by the combined efforts of Hamilton, Perelman, etc.

The important geometric structures found in higher dimensional spaces are those that satisfy the Einstein equations. The importance of Calabi conjecture is that it provides a method to find the geometric structure that 
satisfies Einstein equations in complex geometry. Through such construction we arrive at many interesting conclusions.

These structural theorems are not easy to uncover, but they are crucial to understand geometry. They allow us to provide new geometric tools based on topological information only.

Why do we need to understand the construction of geometric structures? Well, the most direct way to understand a machine is to disassemble and reassemble the entire machine. The most direct way to know a car is to create it from the very steel with which it is built. We must learn how to construct the whole structure undergirding geometry if we wish to truly understand it. This is precisely why existence theorems play such a crucial role in geometry. We can understand the profound and difficult aspects of geometry through these existence theorems.

A beautiful and powerful geometric formula was proven in the 20th century by the three great mathematicians Fritz Hirzebruch, Michael Atiyah, and Isadore Singer. The importance of this result lies in its ability to provide the existence conditions of solutions to differential equations, which allows us to solve many of the problems we are faced with in structural geometry. The work became very effective in the hands of Kodaira, Hirzebruch, Atiyah, Singer, Raoul Bott, Nigel Hitchin, Simon Donaldson, and Edward Witten. The existence of solutions of differential equations was connected to the data of algebraic topology through this formula. It combines both the algebra and the analysis associated with the space.

We have seen that geometric structures may be pretty, but we often forget that, to build these structures, we need to perform thorough analyses of the structures, which may depend on deep a priori estimates of the differential equations involved. Pretty geometric structures need vast amounts of different knowledge to be assembled, just as building a car requires the production of all sorts of different parts.

\section{Conclusions}

Throughout history, most mathematicians have made important contributions while in their youth. Alas, aging is a constraint imposed by our body. When we are young, we are full of energy and we are able to concentrate when solving problems. We need to hold enthusiasm for the object of the knowledge that we pursue, just like the heroes described in Homer's epics, who must persist through great suffering in order to keep moving forward and achieve their goal; and just like the legendary monk Xuan Zang in the 7th century, who embarked on a long journey to the west (in fact, to India) in search of the Buddhist classics. He demonstrated remarkable perseverance in order to achieve success. Without such determination and perseverance, success would be just a dream.
The ancient Chinese poet Qu Yuan said: "having been born with this inner beauty, I adorn it with acquired accomplishment."

And these are the words of Jia Yi, a scholar in the early Han dynasty: "Heaven and Earth are the furnace; the Creator, the smelter; Yin and Yang are the charcoal; living creatures are the bronze."

Without suffering through the fire, we cannot attain great knowledge.

There are many scholars who study very hard, but ultimately cannot make great accomplishments because they do not have a global view of what they are looking for. In much the same way, there are vast numbers of young scholars who like to contemplate philosophy and think highly of themselves, but who accomplish little in mathematics because they do not have the refined technology needed to solve important problems. Therefore, we must be very careful in our learning, and spend plenty of time thinking and debating. Scholars need free will and the spirit of independence to pursue their own ideals and achieve truly great knowledge.

The Emperor of Wei, Cao Pi, in "A Discourse on Literature" from his Authoritative Discourse, remarked that:

The ancients valued not huge pieces of jade disks, but every inch of time, for fear of its creeping by. Yet people usually do not exert themselves: when in poverty and inferiority, they are intimidated by hunger and cold; when amid wealth and honor, they indulge themselves in comfort and pleasure. They are blinded by what lies before their eyes while they blunder away an everlasting accomplishment in history.

There are scholars who fear cold and hunger, and there are scholars who indulge themselves in comfort and ease. Both of them look only at the things around them and forget that the knowledge is built upon the contributions of thousands of years.

How can we work hard to pursue the true meaning of knowledge, without being confused by the temptations surrounding our lives? How can we become personifications of the great men or the holy goddesses depicted in Greek mythology, within the field of knowledge?

In his book, my father quoted these words from The Literary Mind and the Carving of Dragons:

Pity those philosophers! Although they lived in adverse times, they were able to give expression to their ideals in their investigations of the Dao. Their minds reached back to the distant past, their aspirations have resounded throughout the ages. Gold and precious stones may be destroyed, but a great name lives forever.

The time of ancient Greece has passed, but the spirit of seeking truth, beauty and harmony is still worthy of admiration. In this new century, our observations and understanding of the nature will become much deeper than ever before. Can we establish a new trend of thought 
and propel our minds thousands of years into the future? I think this is a question well worth pondering.

\section{Questions taken at the conclusion of the lecture}

\section{Question}

Throughout your lecture, you talked about how one should conduct research. The theoretical developments you mentioned seem so easy, and make for rather smooth reading; however, it's actually accomplished with painstaking efforts and tremendous concentration, which you mentioned once again near the end of the lecture. When you spoke of Riemann's groundbreaking thoughts in his theoretical developments, it sounded like these breakthroughs are necessary in many aspects of mathematical development, and you implied that once one thinks a problem through enough, they will uncover the key to the problem.

My first question is: Is it correct to say that one who takes enough time and concentration will surely succeed in academics?

My second question is: If concentration is essential, we need to be physically strong in the first place, but given that one has a strong body, will they definitely be successful? To put it another way, based on previous mathematical theories, which are generally well known, can one come up with new theories after enough efforts? In reality, it seems like only a few mathematicians could do so.

\section{Answer}

I spoke about history a moment ago-from the five axioms of the Greek development of Euclidean geometry to the birth of Riemann which happened to be right after the birth of the theory of hyperbolic geometry. Riemann may recognize the importance of hyperbolic geometry, but history, however, does not reveal anything to us in this regard. In fact, it was his teacher, Carl Friedrich Gauss, who came to this realization and was among the founders of hyperbolic geometry. At the same time, Gauss proved an incredibly important theorem, which he named the Theorem Egregium (Remarkable Theorem). On the basis of that theorem, he was able to introduce the concept of intrinsic curvature. From there, the idea of Riemannian space would still not enter Riemann's thoughts until after he had spent tremendous amounts of time deliberating the ideas involved. After taking notice of Gauss's theorem, Riemann pondered over the significance of space from a physical standpoint, and this was an idea that could not be developed overnight.

The first new idea to emerge was that of hyperbolic geometry, and it was the Theorema Egregium that lead to Riemann's brilliant idea and the subsequent developments. After this new concept had been created, the discipline itself could be developed comparatively rapidly.
Take the concept of parallelism, as another example. After two thousand years of pondering over the concept of "parallel," the deep theory of gauge fields came into being. Since then, the mathematics and physics involved have been developed very quickly during the 20th century.

Although Riemann was a truly great mathematician, he received major clues from his teacher Gauss. Such is the case with each mathematical research topic, which makes significant contributions-it is gradually built upon the work of countless predecessors. Rome was not built in a day. Gauss already knew that there was something more intrinsic about space, as uncovered in his notion of curvature. This was before Riemann introduced the underlying geometry, to be sure, but Gauss failed to come up with the concept of inner space in order to describe the absolute curvature. It was Riemann who finally unmasked the spatial structure hidden beneath.

The whole process is not quite as one may have imagined, i.e. that one morning a new idea will occur to you, and you will succeed academically immediately afterward. This is usually not the case. Typically, you have to spend a significant amount of time critically thinking about the problem and concentrating on studying it. That is the reason that I placed health as such a high priority-this is the only way to improve concentration-and, yet, health is not the sole factor determining success. If one can concentrate, however, they can avail themselves of time to think about, and learn more about, the problem at hand. Generally speaking, it takes three or more years to obtain a great achievement. It is difficult to make it happen in less than three years of hard work.

\section{Question}

You just mentioned that mathematicians are usually successful when they are young. In other disciplines, things seem to be quite the opposite. Can you explain the differences?

\section{Answer}

Both mathematicians and physicists are scientists who work on theories. Generally speaking, they are capable of better employing their creativity when they are young. As for the engineers, their work is usually carried out by, and finished by, a large group of contributors, rather than by oneself; thus, an individual's creativity is not the only determining factor. In theoretical sciences, however, most accomplishments are made independently, or through the cooperation of a comparatively small group. Many breakthroughs in the theoretical sciences require quite novel ideas, which are created by a small group of people, each with full concentration. It is primarily in this way that the importance of youth enters into these fields. One more advantage possessed by younger researchers is that they are slightly less burdened by traditional thoughts, and they spend less time on administrative and social activities compared to more senior researchers. This was why Einstein and Newton 
were able to make their most important contributions before the age of 30. Most physicists and mathematicians achieve great things when they are young.

\section{Question}

Compared with the entire history of human civilization, I'd like to know your opinions on the historical progress of mathematics during the past one or two centuries. If we use a curve to represent the progress, what kind of curve do you think is suitable? Would it be an exponential curve? If not, I was wondering if you could offer your views on the future development of mathematics to be carried out by a new generation of young mathematicians, based on your observations. That's the first question. The second question is on a slightly different topic. Historically, fierce competition exists in both physics and mathematics. What kind of impact do you think the progress in fields such as physics will have on mathematics? Will mathematical development be sped up?

\section{Answer}

Mathematics has undergone great development in the past century. There is one important feature of this time period, however, that we should first recognize: the number of people engaged in mathematical studies is substantially larger than before. If we were to combine all of the middle school students from Mainland China and Taiwan, that would nearly be 10 million people. The best among the 10 million are selected and move on into universities. From there, the best researchers in these universities are picked. It was impossible to make this happen for such a large number of people during the ancient times, due to their comparatively small population. This huge number of people can be very helpful, but it is not quite so easy to identify the most talented, or to provide them with appropriate training. This is why countries of smaller size, such as Netherlands and Hungary, are able to produce numerous prominent mathematicians-culture and tradition can be of considerable help, which also arose as the result of many years of research.

Therefore, apparently there are more advantages in the world of mathematics available today compared to before. In spite of this, there are still some strong features of research in the past. It is hard to make a comparison.

Not only that, but it is hard to judge whether an exponential curve could be used to describe the most recent progress. Mathematicians have obtained great achievements in the 300 years following the Renaissance and the discovery of calculus. Indeed, I just recently calculated that there are about one or two hundred mathematicians possessing great creativity from the 18th to the 19th century. Their important contributions are no worse than that of mathematicians in the present century. During today's lunch, I spoke with academician Peng about Leonhard Euler and his work. We agreed that even up to now mathematicians had not been able to put together a complete picture of Euler's work. Euler was a mathema- tician in the 18th century whose work was truly monumental. It is difficult to find mathematician, in the present century that can be compared with him. Consequently, we can hardly say that mathematics has been developed at an exponential rate compared to the great accomplishments made by ancient mathematicians.

There is no doubt that much important scholarly progress has been made in this century. I suppose that the entirety of the contributions made these days is no worse than before, and they have been growing all the time. In my opinion, mathematics, especially pure mathematics, has made genuinely significant progress, with the solution of many major problems. I don't believe that mathematical developments will slow down. I predict that mathematics will maintain a constant rate of development in the future.

Mathematicians have cultivated close connections with physicists throughout the past four decades. We have had a tremendous influence on physicists, who have, in turn, had an impact on us. It has been quite a healthy development, which will certainly continue. I suppose we are bound to see much more beautiful prospects in mathematics in the coming century.

\section{Question}

You just mentioned harmony, which led me to think about a question. First, please allow me to clarify one thing. It's about the Archimedean dodecahedron. Is it really worth figuring out the solution to a twelfth-order equation?

\section{Answer}

It makes some sense to get to know more about a special equation through calculations. Many special equations are perfectly sensible; however, we need the theoretical framework underpinning them to express their true meaning. Figures themselves may not have much significance in and of themselves, but we need to understand their underlying significance. Consider calculations as an example: even if we work out millions of digits in a calculation, we may not know all that much about the problem in which it arose. Calculation surely has its importance in the understanding of an equation, but that is definitely not the sole factor. The key factor is, in fact, to develop a deep understanding of the equation, rather than the mere digital understanding provided by a calculation.

\section{Ouestion (continued)}

What I mean is that if the problem is pursued via equations, it may result in a twelfth-order equation. In contrast to this, through geometric methods we can actually calculate a square root or a cubic root, which might involve a very complex formula, and in this way we're sure to get a precise value.

\section{Answer}

It would certainly be great to find the so-called "closed form" solution to any given problem. Sometimes, 
however, we can simply use a calculator to figure it out, but it may not be the most important aspect to work on. Our focus is on getting to know the whole equation and uncovering its algebraic, analytic, or geometrical significance. It is our hope that new branch of sciences can be developed on the basis of the framework unearthed in this manner.

The parallel axiom I mentioned today seems unimportant. It was found, however, that based on this axiom, new disciplines in geometry was developed. This discovery turns out to be far more important than the parallel axiom itself.

Let me give you another example. Fermat's Last Theorem is a well-known problem; however, it didn't attract great interest among "professional" number theorists in the first half of last century until the beginning of the last half century, for it was seemingly irrelevant to other problems until that time. After Andrew Wiles' proof of the theorem, its importance became clear. The reason for this is that his proof incorporates numerous important branches in number theory. The importance of the development of those branches is greater than that of Fermat's Last Theorem itself. In this way, we see that it is much more about the way in which we view the issues at hand.

\section{Question}

You mentioned that every middle school student should learn plane geometry, which seems to be underrated in modern times. It seems that experimentalism has had great influence on that. Would you please talk about this?

\section{Answer}

I think that plane geometry is a very important subject. It is not necessarily the beautiful and significant theorems that are important, but rather the reasoning process used to obtain them. A great way for middle school students to learn logic is through plane geometry, from which they can also develop an appreciation for the beauty of geometry itself. It is the reasoning process that I want to stress though. I think that middle school students should learn plane geometry, and through this they will learn how to prove theorems and understand syllogisms.

\section{Question}

You mentioned the beauty inherent in symmetry. In actuality, asymmetry can also possess its own beauty sometimes. When it comes to this concept, how can we carry out research using geometry? Symmetry seems useful in light of the intuitive feeling provided by physics, namely the transformation of energy and the notion of Ricci flow just mentioned, but, can asymmetry also play a role? For example, is there a notion of asymmetric entropy? Are there any clues or indications of this in your research?

\section{Answer}

I spoke a lot about the role played by the symmetric group in geometry and physics. Most times, however, many geometric objects we consider have no symmetry, which means that there is no obvious symmetry group or groups. Some objects, though, do have obvious symmetry group or groups, such as Platonic solids mentioned before. In order to resolve this disparity between objects possessing obvious symmetry and those that do not, geometers introduced the concept of internal symmetry. Space itself may not have external symmetry, but it may have internal symmetry. The concept of gauge fields was developed from this concept of internal symmetry. As for the concept of entropy, it arises within the physics of dynamical systems. When we were studying dynamical geometry, the concept of entropy also exists and it should have something to do with entropy in quantum mechanics. The important concept of entropy also plays a role in our research on Ricci flow as well. That being said, however, I'm afraid that I cannot give detailed explanations on this matter today, since time is limited. 